\title{
MICRO-ALBUMINIURIA AS A PREDICTOR OF LEFT VENTRICULAR HYPERTROPHY IN NON-DIABETIC HYPERTENSIVES
}

Satish Kinagi ${ }^{1}$, Deepak Bhanukumar ${ }^{2}$, Naveen Golabavi3 ${ }^{3}$ Naganagouda Patil ${ }^{4}$

${ }^{1}$ Associate Professor, Department of Medicine, MRMC, Kalaburagi.

2 Post Graduate, Department of Medicine, MRMC, Kalaburagi.

${ }^{3}$ Assistant Professor, Department of Medicine, Belagavi Institute of Medical Sciences, Belagavi.

${ }^{4}$ Senior Resident, Department of Medicine, St. Johns Medical College, Bangalore.

\section{ABSTRACT}

\section{BACKGROUND}

Microalbuminuria is an early manifestation of kidney damage and independently predicts cardiovascular disease (CVD). Microalbuminuria is considered a reliable indicator of endothelial dysfunction. Endothelial dysfunction is considered an early step of atherosclerosis and represents increased risk of cardiovascular events. Left ventricular hypertrophy (LVH) is also an early marker of cardiac manifestation of target organ damage among hypertensive patients. This study aimed at determining microalbuminuria as an early predictor of left ventricular hypertrophy in non-diabetic hypertensives.

\section{AIMS}

To identify left ventricular hypertrophy in essential hypertensive patients and correlation of left ventricular hypertrophy with microalbuminuria in hypertensive individuals.

\section{DESIGN}

Cross-sectional study.

\section{MATERIALS AND METHODS}

One hundred patients of essential hypertension with or without left ventricular hypertrophy who were non-diabetics were studied over a period of 2 years. Urine PCR, ECG, 2D Echo were performed and Urine Microalbuminuria was correlated with left ventricular mass index.

\section{RESULTS}

It is observed that out of 100 patients, 56 patients were having proteinuria, 45 were having microalbuminuria and 11 had macroalbuminuria.49 had abnormal cardiac geometry in the form of left ventricular hypertrophy in hypertensive patients.

\section{CONCLUSION}

There is significant association between LVH and microalbuminuria in hypertensive patients. Thus, we can recommend microproteinuria as the screening procedure for target organ damage in hypertensive patients so as to select our treatment strategies accordingly.

\section{KEYWORDS}

Microalbuminuria; Left Ventricular Hypertrophy; Cardiac Geometry; Target Organ Damage.

HOW TO CITE THIS ARTICLE: Kinagi S, Bhanukumar D, Golabavi N, et al. "Micro-albuminuria as a predictor of left ventricular hypertrophy in non-diabetic hypertensives." Journal of Evolution of Medical and Dental Sciences 2015; Vol. 4, Issue 105,

December 31; Page: 17023-17026, DOI: 10.14260/jemds/2015/2575

\section{INTRODUCTION}

Affecting one billion people worldwide systemic hypertension remains the most common, readily identifiable and reversible risk factor for myocardial infarction, stroke, heart failure, atrial fibrillation, aortic dissection and peripheral arterial diseases. The global burden of hypertension is projected to affect 1.5 billion persons, one third of world's population, by the year 2025.1,2 Cardiovascular disease risk doubles for every $20-\mathrm{mmHg}$ systolic and $10-\mathrm{mm} \mathrm{Hg}$ diastolic rise in blood pressure. $^{3}$

Financial or Other, Competing Interest: None.

Submission 30-11-2015, Peer Review 02-12-2015,

Acceptance 26-12-2015, Published 30-12-2015.

Corresponding Author:

Dr. Satish Kinagi,

Department of Medicine,

Basaweshwar Teaching and General Hospital,

Sedam Road,

Kalaburagi-585105.

E-mail: deepak.b.jssmc@gmail.com

DOI:10.14260/jemds/2015/2575
WHO, ESH guidelines have indicated the following manifestations of target organ damage as factors influencing prognosis. ${ }^{4}$

1. Left ventricular hypertrophy.

2. Radiological evidence of atherosclerotic plaque.

3. Proteinuria, slight elevation of plasma creatinine concentration.

4. Generalized focal narrowing of the retinal arteries.

Increased left ventricular mass or Left Ventricular Hypertrophy (LVH) is a frequent complication of hypertension. This condition is another manifestation of preclinical disease and has long been known as a powerful independent risk factor for all of the cardiovascular complications of hypertension and also has been associated with increased morbidity and mortality. ${ }^{5-6}$ Identification of various geometric patterns of LV hypertrophy further stratifies cardiovascular risk. ${ }^{7-8}$

Microalbuminuria is a specific integrated marker of cardiovascular risk and target organ damage in primary hypertension and one that is suitable for identifying patients at higher global risk.9,10 Microalbuminuria is considered as 
strong, early and independent marker of increased cardiovascular risk in hypertension. ${ }^{11}$

In hypertensive patients, Microalbuminuria has significant association with concentric and eccentric LV hypertrophy. ${ }^{12}$ Urine albumin creatinine ratio positively correlates with LV mass, systolic BP, age, pulse pressure and endocardial and mid-wall shortening, but not to diastolic filling parameters. ${ }^{12}$ Micro-albuminuria confers a fourfold increased risk of Ischemic heart disease among hypertensive or borderline hypertensive subjects. ${ }^{13}$

Microalbuminuria is considered a reliable indicator of endothelial dysfunction, this being associated with transendothelial escape of albumin and plasma levels of von Willebrand factor. ${ }^{14-15}$ Microalbuminuria is indeed correlated with endothelial dysfunction in the brachial artery. ${ }^{16}$ Also endothelial dysfunction is considered an early step of atherosclerosis and represents increased risk for CV events. ${ }^{17}$ Micro-albuminuria is also associated with impaired aortic mechanics and increased LV mass. ${ }^{18-22}$ Proteinuria, in hypertensive renal disease. ${ }^{21-24}$ may accelerate the decline of renal function and also amplify the risk of vascular disease. Losartan Intervention for end point reduction in hypertension study (LIFE study) indicates that changes in urine albumin excretion under antihypertensive treatment parallel those of ECG determined LV mass. Determination of albuminuria can become a useful tool in evaluation of global cardio vascular risk. $^{25}$

\section{METHODOLOGY}

Study Method

Cross-sectional Study.

\section{Subjects and Methods}

By simple random study, 100 patients of essential hypertension with or without left ventricular hypertrophy were taken with all the exclusion criteria and studied over a period of 2 years.

\section{Inclusion Criteria}

1. Patients with systolic BP $>140 \mathrm{mmHg}$ and/or diastolic BP $>90 \mathrm{mmHg}$ in at least 2 visits.

2. Age more than 40 years.

3. No clinical or lab evidence of heart failure, renal failure, coronary artery disease, valvular heart disease, ischemic cardiomyopathy secondary hypertension, diabetes mellitus, hyperlipidemia, overt proteinuria, urinary tract infection, fever or exercise.

In all these patients history of substance abuse, comprehensive clinical examination and appropriate imaging and bio-chemical evaluation done.

\section{Blood Pressure}

BP recordings were taken with well-calibrated mercury sphygmomanometer and proper cuff size and staging was given as stage I:140-159/90-99.

Stage II: $>160 />100$.

\section{Electrocardiogram}

\section{Sokolov-Lyon Voltage Criteria}

In 1949, Sokolov and Lyon pointed out that the presence of left ventricular hypertrophy in adult is suggested when the sum of $\mathrm{S}$ wave in $\mathrm{V} 1$ and $\mathrm{R}$ wave in V5 or V6 potentials are more than $35 \mathrm{~mm}$.

\section{Echocardiographic Examination}

Echocardiographic examination was performed at Department of Cardiology by a cardiologist, unbiased of patient status. Two-dimensional M-mode echocardiography was done, standard parasternal and apical view observed with patient supine in left lateral position.

\section{Proteinuria Measurement}

The morning spot urine sample, protein-creatinine ratio has been measured using turbidimetric method (sulphosalicylic acid method) after ruling out overt proteinuria and cellular deposits. Positive- Urine PCR $>30 \mathrm{mg} / \mathrm{g}$ of Creatinine. Negative-Urine PCR $<30 \mathrm{mg} / \mathrm{g}$ of Creatinine.

\section{RESULTS}

Majority of patients in this study group were in fifth decade of life $(46 \%)$. The study group consisted of 58 males and 42 females. Sex ratio male-to-female is 1.38:1.

Distribution of patients with hypertension according to JNC-7 classification

\begin{tabular}{|c|c|c|}
\hline Stage of Hypertension & $\begin{array}{c}\text { Number of } \\
\text { Patients }\end{array}$ & Percentage \\
\hline Stage-I $(140-159) /(90-99)$ & 35 & 35 \\
\hline Stage-II $(\geq 160 / \geq 100)$ & 65 & 65 \\
\hline Total & $\mathbf{1 0 0}$ & $\mathbf{1 0 0}$ \\
\hline
\end{tabular}

Distribution of patients according to pulse pressure gradient

\begin{tabular}{|c|c|c|}
\hline Pulse Pressure & $\begin{array}{c}\text { Number of } \\
\text { Patients }\end{array}$ & Percentage \\
\hline$<40$ & 18 & 18 \\
\hline $41-50$ & 36 & 36 \\
\hline $51-60$ & 29 & 29 \\
\hline$>60$ & 17 & 17 \\
\hline Total & $\mathbf{1 0 0}$ & $\mathbf{1 0 0}$ \\
\hline $\begin{array}{c}\text { Mean } \pm \text { SD of pulse } \\
\text { pressure }\end{array}$ & \multicolumn{2}{|c|}{$\mathbf{5 1 . 9 8 \pm 1 1 . 4 6}$} \\
\hline
\end{tabular}

Distribution of patients according to urine proteincreatinine ratio in proteinuria range

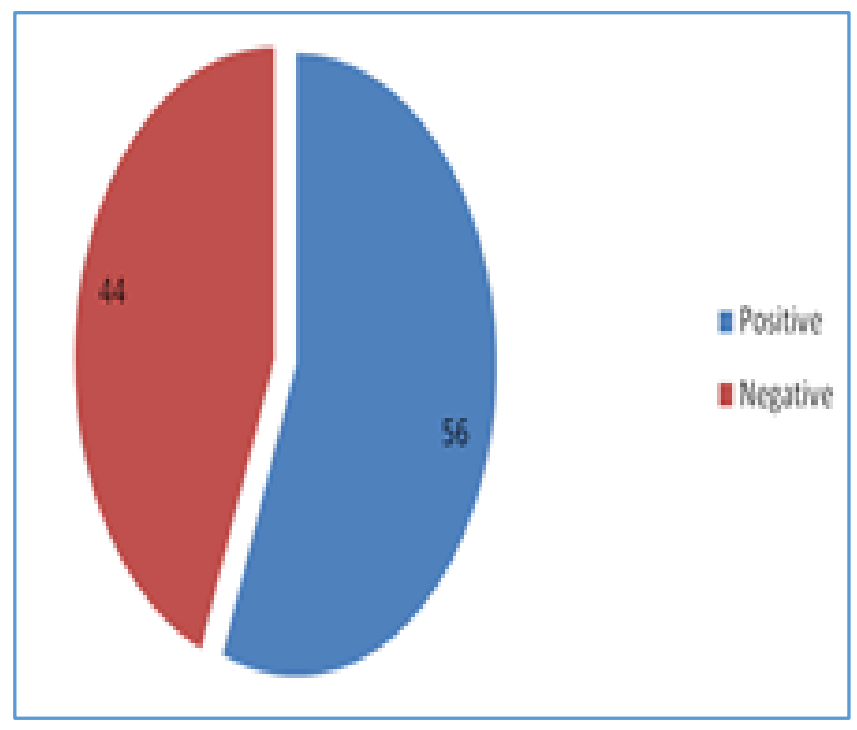

Association between Urine protein-Creatinine Ratio and Abnormal Cardiac Geometry

\begin{tabular}{|c|c|c|c|c|c|c|}
\hline \multirow{2}{*}{ Proteinuria } & \multicolumn{3}{|c|}{ Cardiac Geometry } & \multicolumn{2}{|c|}{ Total } \\
\cline { 2 - 7 } & \multicolumn{2}{|c|}{ Abnormal } & \multicolumn{2}{c|}{ Normal } & \multicolumn{2}{|c|}{} \\
\cline { 2 - 7 } & No. & Percent & No. & Percent & No. & Percent \\
\hline Microproteinuria & 36 & 80.00 & 9 & 20.00 & 45 & 45.00 \\
\hline Macroproteinuria & 7 & 63.63 & 4 & 36.36 & 11 & 11.00 \\
\hline Normal & 6 & 13.63 & 38 & 86.36 & 44 & 44.00 \\
\hline Total & $\mathbf{4 9}$ & $\mathbf{4 9 . 0 \%}$ & $\mathbf{5 1}$ & $\mathbf{5 1 . 0 0}$ & $\mathbf{1 0 0}$ & $\mathbf{1 0 0 . 0 0}$ \\
\hline
\end{tabular}

$\chi^{2}=40.267 \mathrm{P}<0.0001$ Very highly significant. 


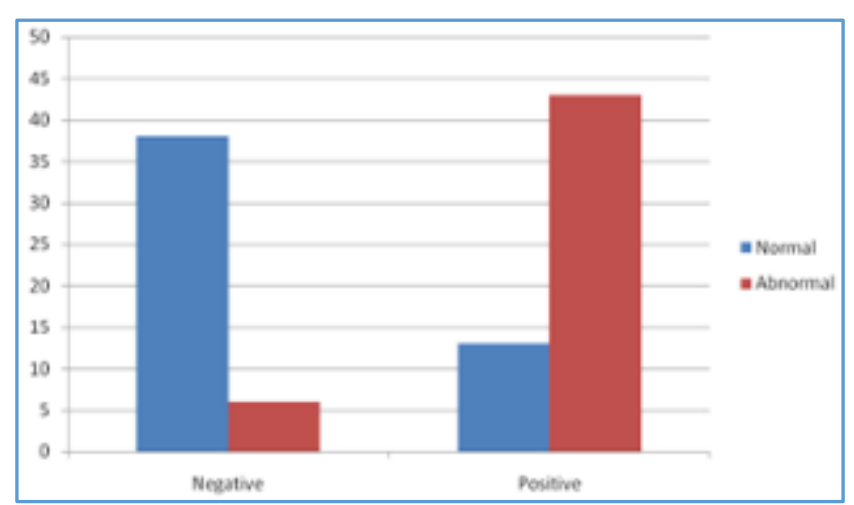

Association between Stage of Hypertension and Urine protein creatinine ratio

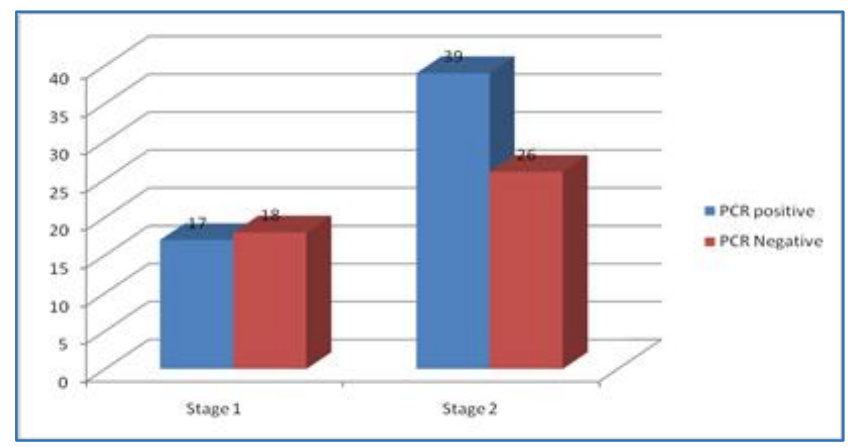

Among 56 patients with proteinuria, 39 (60\%) patients in stage II hypertension had proteinuria, whereas only 17 patients in stage 1 hypertension had proteinuria. Thus the study shows that stage-II hypertension patients had more proteinuria when compared to stage 1 hypertensive patients.

\section{Association between urine PCR and cardiac geometry in} patients with stage II Hypertension

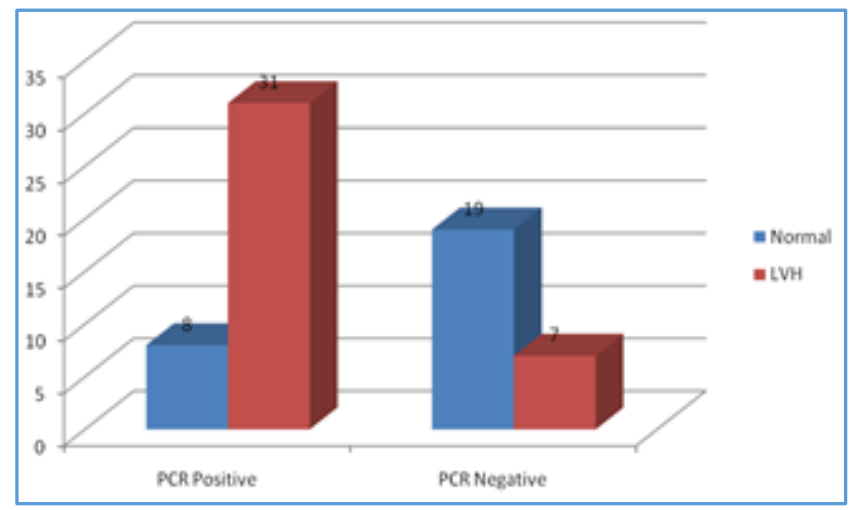

Thus the study reveals that among 65 patients with stage II hypertension, 31 patients had LVH of which 39 patients had proteinuria. Thus it shows that patients with stage 11 hypertension have more proteinuria and LVH.

\section{DISCUSSION}

Study was conducted in 100 essential hypertensive patients in the age group of more than 40 years of age with $58 \%$ males and $42 \%$ females. Majority of the patients were in $50-59$ years of age group. As per JNC-7 staging of hypertension, 35 (35\%) of patients were in stage I and $65(65 \%)$ were in stage -II.

When protein creatinine ratio and abnormal cardiac geometry was correlated, the study revealed that there is strong association between microproteinuria and LVH in patients with essential hypertension.

A significant association was also seen between widened pulse pressure and abnormal cardiac geometry. Incidence of LVH was more in patients with widened pulse pressure. $62 \%$ with pulse pressure in the range of 51- 60 and $70 \%$ with pulse pressure more than 60 had LVH respectively, while majority of patients in the pulse pressure range of $<40$ and 41-50 had normal geometry. Thus patients with wide pulse pressure had more abnormal cardiac geometry. BMI showed significant association with proteinuria and LVH.

Among 35 patients with stage I hypertension 57\% had normal cardiac geometry and $40 \%$ had microproteinuria. And among 65 patients with stage II hypertension, 58\% had LVH of which $60 \%$ had microproteinuria. Thus stage 11 hypertension patients had more proteinuria and more association with $\mathrm{LVH}$. Microalbuminuria is an early predictor of endothelial dysfunction and ongoing atherosclerosis. It is a reliable predictor of cardiovascular and cerebrovascular morbidity and mortality. Hence, it can be recommended that, microproteinuria as a screening procedure for target organ damage in essential hypertensive patients so as to select our treatment strategy accordingly.

\section{CONCLUSION}

The study has shown a significant association between LVH and microproteinuria in hypertensive patients; in conformance with different studies as quoted above. The study also shows significant association of microproteinuria with wide pulse pressure, stage II hypertension and BMI. Microalbuminuria is an early predictor of endothelial dysfunction and ongoing atherosclerosis. It is a reliable predictor of cardiovascular and cerebrovascular morbidity and mortality. Hence, it can be recommended that, microproteinuria as a screening procedure for target organ damage in essential hypertensive patients so as to select our treatment strategy accordingly.

\section{BIBLIOGRAPHY}

1. Theodore A Kotchen. Harrison's Principles of Internal Medicine 19th ed., volume 2, 2015, McGraw Hill Education, chapter 298, page no 1612.

2. Chobanian AV, Bakris GL, Black HR, et al. The seventh report of joint national committee on prevention, detection, evaluation and treatment of high blood pressure: The JNC 7th report JAMA 289:2560, 2003.

3. Vasan RS, Massaro JM, Wilson, et al. Antecedent BP and risk of cardiovascular disease; Framingham Heart Study Circulation 2002;105:48.

4. Kannel and Abbott RP. A Prognostic comparison of Asymptomatic LVH and unrecognized MI. The Framingham study, Annual Heart Journal Volume. III, 391 397, 1986.

5. Tsioufis C, Stefanadis C, Toutouza $M$, et al Microalbuminuria is associated with unfavourable cardiac geometric adaptations in essential hypertensive subjects. Journal of Human Hypertension. 2002;16:249-4.

6. Wachtell $\mathrm{K}$, Palmieri $\mathrm{V}$, Olsen $\mathrm{MH}$, et al. Urine albumin/creatinine ratio and echocardiographic left ventricular structure and function in hypertensive patients with electrocardiographic left ventricular hypertrophy: The LIFE study. Am Heart J 2002;143:319-26.

7. Pontremole R, et al. Prevalence and clinical correlates of microalbuminuria in essential hypertension. AGIC study hypertension 1997;30:1135-1143.

8. Cerasola G, et al. Microalbuminuria renal dysfunction and cardiovascular in complication essential Hypertension J. Hypertension 1996;14:915-920. 
9. Pontremole R, et al. Microalbuminuria, $\mathrm{CV}$ and renal risk in primary hypertension. JAN Soc Nephrol 13:s169-s172, 2002.

10. Viberti G, et al. should we screen for microalbuminuria in essential hypertension? Am J Kidney Dis 1999;34:11391141.

11. Bohm $\mathrm{M}$, Thoens $\mathrm{M}$, et al. Association of $\mathrm{CV}$ risk factors with MAG in hypertension J. Hypertension, 2007 Nov, 25:2317-24.

12. Greene et al. Microvascular rare faction and tissue. Vascular resistance and hypertension. Am J Physiology 256:1t126-131, 1989.

13. Jensen JS, et al. Arterial hypertension, Microalbuminuria and risk of ischemic heart disease hypertension 2000;35:898-903.

14. Deckert T, Feldt-Rasmussen B, Borch-Johnsen K, et al. Albuminuria reflects widespread vascular damage. The steno hypothesis. Diabetologia 1989;32:219-26.

15. Pedrinelli R, Giampietro 0 , Carmassi F, et al. Microalbuminuria and endothelial dysfunction in essential hypertension. Lancet 1994;344:14-8.

16. Stehouwer CD, Henry RM, Dekker JM, et al. Microalbuminuria is associated with impaired brachial artery, flow-mediated vasodilation in elderly individuals without and with diabetes: further evidence for a link between microalbuminuria and endothelial dysfunction the Hoorn Study. Kidney Int Suppl 2004;66:S42-4.
17. Quyyumi AA. Prognostic value of endothelial function. Am J Cardiol 2003;91:19H-24.

18. Borch et al. UAE and Independent Predictor of Ischemic Heart Disease Arterioscler Thromb Vas. Biol 1999;19:1992-1997.

19. Tsouif Cetal. Microalbuminuria in Association with Abnormal Thoracic Aortic Mechanics in Essential Hypertension; Am J Cardkil 2000;86:797-801.

20. Pedrinelli P. Micro-Albuminuria in Hypertension Nephrol 1996;73:499-506.

21. Greene $\mathrm{T}$ Heberti, et al. Magnitude of Proteinuria Reduction and Risk of Renal Disease Arch Intern med 2005;165:247-53.

22. Adler, et al. BP control, Proteinuria and Progression of Renal Disease. Ann Intern med 1995;123:754-62.

23. Mann IF, et al. Albuminuria and Risk of Cardiovascular Events, Death and HF in Diabetic and Non-Diabetic Patients-Jama 2001;286:421-26.

24. Sacchi G, et al. MAU identifies overall Cardiovascular Risk in Essential Hypertension: An artificial Neural Network Approach J Hypertension 2002;20:1315-21.

25. Pontremoli, et al. Cardiovascular and Renal Risk Assessment as guide for Treatment in Primary Hypertension J am soc Nephrol 15;S36-36, 2004. 